\title{
Design and Realization of Supervision and Inspection of Fire System Based on .net
}

\author{
Chenying Yang \\ The Chinese People's Armed Police Forces Academy, Langfang, China \\ y_feiruth@sina.com
}

Keywords: Supervision and inspection of fire, ASP.net, B/S model

\begin{abstract}
With the rapid development of Chinese economic, the effect of supervision and inspection of fire has become more and more important. According with the workflow and requirements of supervision and inspection, this system is from the actual needs of firefighting work, operated easily and is suitable for practical application of fire force. B/S model was adopted in this system and the article was going to be written that based on ASP.net and SQL Server. The system principles, the system function analysis and design, operational procedures, and key technologies realized were explained separately.
\end{abstract}

\section{Introduction}

With the fast development of computer and network technology, computers have been used extensively to the fire control work as an effective way to improve the speed and efficiency ${ }^{[1]}$. The cooperation office automation platform of electronization and netwokization of fire department get the extensive universality. Fire prevention is the most important in fire protection. Relying on digital platform, fire inspection management system can accelerate the speed of the fire inspection data collection, compilation, analysis and information dissemination, which improves the efficiency of data processing, ensures the correctness and completeness of statistics, improves the quality of the legal documents, file management and inspection ${ }^{[2]}$.

Fire Integration information system which be used in Fire forces is a huge scale, multifunctional system and whose original difference is not obvious. In this paper, the design and implementation of supervision and inspection system were researched and discussed relying on the development of a certain place fire inspection system. In system, we divided the subsystem and the modules from the system functional requirements and discussed the design and implementation of parts of the system functions.

\section{System Analysis}

In the fire inspection, the system is mainly used to record the generation and the end of the mission including several modules, for example: supervision and inspection, supervision and review, risk supervision, fire investigations and administrative penalties. Using this system we can complete tasks in time and improve the efficiency of the fire department. Administrators can manage information of users and units, add or delete announcements, view other tasks, send email to remind; besides these the administrator user has certain supervisory functions including setting users' names and passwords. In system, the user's password in the database is encrypted with the MD5 digest ${ }^{[3]}$, which greatly improves the security of the system. System functions as follows:

\subsection{Rights management module}

System privileges are divided into two kinds: administrator and users. The main function of rights management module: login different business process interface according to the scope of duties and activities of the logged in, query user's information and add, delete, and modify user's information, modify user's type and login password. Only super administrators have permission to add, delete and modify a user's types, other users can only modify their own passwords. 


\subsection{Unit Management Module}

The main functions of the unit management module include querying, inputting, modifying and deleting, and exporting units' information in word and excel form.

\subsection{Supervision and inspection module}

Supervision and inspection module generates inspection tasks and review tasks. If the unit is required to be inspected failed to generate the review mission to re-examine, and if the second fails, go to the next module, the administrative penalties module.

\subsection{Risk supervision module}

This module is a module for fire hazards which can be divided into general fire hazards and major fire hazards. Hidden supervision tasks are generated in this module, if not promptly corrected the units can be generated its punishment.

\subsection{Fire Investigation Module}

Fire investigation of supervision and inspection is an important feature. In the module, the details of the fire investigation are established, fire investigation tasks are generated, units or individuals are investigated, punish are generated after the investigation task and go to the next module, administrative penalties.

\subsection{Administrative penalties module}

This module generates a direct punishment task, which is based on several modules of the above.

\section{System Design and Implementation}

\subsection{System operating procedures}

The operation flow is shown in figure 1. Ordinary users have rights of supervision and inspection, risk supervision, administrative penalties. In addition, advanced users can perform system maintenance and other operations.

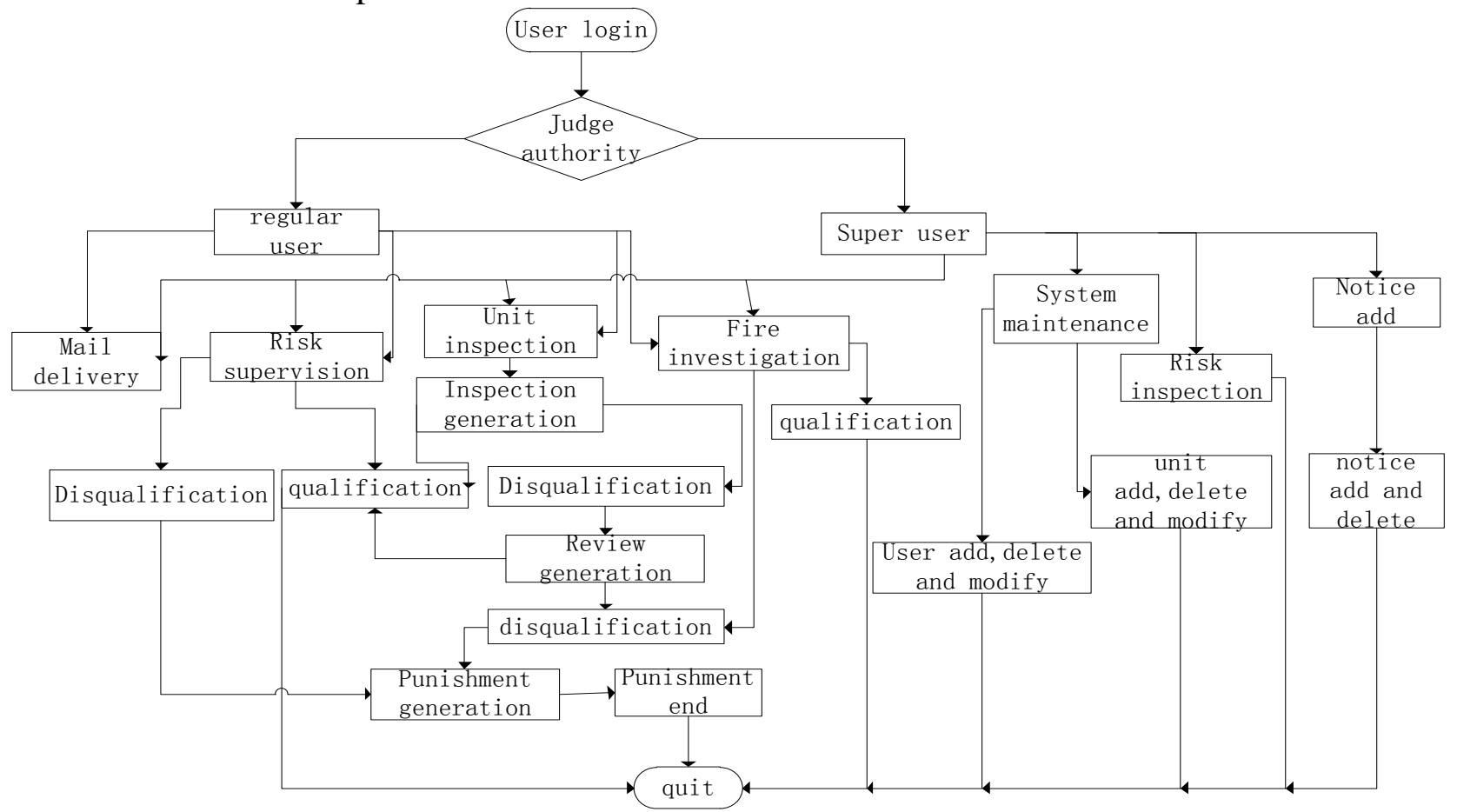

figure1 System operating procedures

\subsection{Key technologies to achieve}

\subsubsection{Realization of module of inspection tasks generated and ended.}

1. Inspection tasks generated

The specific code as follows: 
con.ConnectionString

= ConfigurationManager. ConnectionStrings[FLYconnectionString].ConnectionString;

$\cdots$

cmd.CommandText="insert into JCbiao(danwei,mingcheng,leixing,didian,mianji,zerenren,phone, shixiang,riqi,zhuangtai,fuzheren) values ('" $\quad$ +DW.Text.ToString()+"',"'+MC.Text.ToString()+"',"' +DropDownList2.Text.ToString()+"',"'+DD.Text.ToString()+"',"'+MJ.Text.ToString()+"',"'+ZRR.Text. ToString()+"',"'+DH.Text.ToString()+"',"'+DropDownList1.Text.ToString()+"',"'+TextBox1.Text.ToStri ng()+"',' Processing ',"' + DropDownList3.Text.ToString() + "')";

...\}

2. Inspection tasks ended

After the tasks are ended, the check record sheet needs to be uploaded and the recording sheet will be reflected in the unit information. The specific code as follows:

danwei=Request.QueryString["danwei"].ToString();

if (FileUpload1.PostedFile.FileName == "')

\{

Response.Write("<script>alert(' Please select a file to upload ')</script>");

\}

else

\{

string strPath = this.FileUpload1.FileName;

string vsurl = Server.MapPath(" /JDJC/jianchabiao/") + strPath; // Saving path

cmdd.CommandText = "update JCbiao set zhuangtai = ' Expired ' where danwei ="' + danwei + "'";

cmdd.ExecuteNonQuery();

cnn.Close();

\}

3.2.2 Realization of module of the unit information

The specific code as follows:

string no= Request.QueryString["no"].ToString();

SqlConnection con = new SqlConnection();

..

$\{\ldots$

private void CreateBulletinLayout(SqlConnection cnn, Table tbl, int topN)

if (dr.HasRows)

\{

while (dr.Read())

\{

string tmp = "';

newRow = new TableRow ();

newCell = new TableCell();

newCell. VerticalAlign = VerticalAlign.Top;

newRow.Cells.Add(newCell);

newCell = new TableCell();

tmp = dr["jianchabiao"].ToString();

newCell.Text $=$ "<a href='jianchabiao" + "/" + dr["jianchabiao"].ToString() + " 'target='_blank' $><$ font

color=\#66666 $>$ " + tmp.Substring $(0$, tmp.Length $)+$ "</font $></ a>"$;

Select the path to open the file

$\ldots\}$

\}

tbl.BorderStyle $=$ BorderStyle.None;

dr.Close();

\} 


\section{Conclusions}

Using the system, we can systematically register and manage Fire Brigade file, fire jurisdiction, firefighters, etc., and the management of fire inspection institutionalized and standardized can be achieved, system is targeted. The system which provides a comprehensive information management institution makes the fire agency personnel have a better grasp to supervision and inspection work within the jurisdiction, which will help the progress of work for firefighters. The system uses .net platform and B / S mode ${ }^{[4,5]}$, highlights in information sharing and can provide a better tool for the management of the area-wide unit.

\section{References}

[1] Denggao Zeng: NET system architecture and development. Beijing, Publishing House of electronics industry, vol.1(2003).

[2] Jun Bao: Status and development research of fire supervision and inspection. Value Engineering, vol.4(2014), p.296-297.

[3] Zong chao Lin: Application of the MD5 algorithm based on C\#. Journal of Changchun University of Technology(Natural Science Edition), vol.32(2011), p.581-583.

[4] Yang Zhang: Design and research journal manuscript man-agement system based on .NET. Journal of Southwest Uni-versity for Nationalities(Natural Science Edition), vol.38(2012), p.982 $-986$.

[5] Xiang Ma: Workflow approval system design and imple-mentation based on .NET. Computer Engineering and De-sign, vol.33(2012), p.4187-4257. 